\title{
Grain Boundary Traction Signatures: Quantifying the Asymmetrical Dislocation Emission Processes under Tension and Compression
}

\author{
Ruizhi Li, Huck Beng Chew* \\ Department of Aerospace Engineering, University of Illinois at Urbana-Champaign, Urbana, IL, \\ USA
}

*Author to whom all correspondence should be addressed. Email: hbchew@illinois.edu

\begin{abstract}
The disruption in crystallographic arrangement of atoms across a grain boundary interface generates local stress fields in the vicinity. Here, we reconstruct the continuumequivalent grain boundary tractions from local atomic stresses near symmetrical-tilt $\langle 110\rangle \mathrm{Ni}$ grain boundaries. We show that the resolved shear stress contribution from the grain boundary tractions, $\tau_{\mathrm{GB}}$, along active slip-systems either assists or prevents the emission of dislocations, depending on its direction with respect to the resolved shear stress contribution from external loading, $\tau_{\mathrm{ext}}$. When $\tau_{\mathrm{GB}}$ acts in the same direction as $\tau_{\mathrm{ext}}$, Shockley partial dislocations are readily emitted from the boundary once $\left|\tau_{\mathrm{GB}}+\tau_{\mathrm{ext}}\right|$ exceeds the critical barrier stress for shearslip. When $\tau_{\mathrm{GB}}$ opposes $\tau_{\mathrm{ext}}$, the higher sustainable stresses in the grain boundary structure instead triggers: (a) emission of dislocations from the bulk, or (b) reconfiguration of the grain boundary atomic structure and subsequent emission of non-Schmid dislocations or formation of extrinsic stacking faults. Our results quantitatively explain the asymmetrical grain boundary dislocation emission processes observed in molecular dynamics (MD) simulations under applied tensile and compressive loads. The relationship between the traction signatures and periodic structural units along the grain boundary is discussed.
\end{abstract}

Keywords: Grain boundary tractions; dislocation emission; tension-compression asymmetry; molecular dynamics; nanocrystalline metals 


\section{Introduction}

Nanocrystalline metals have ultra-high strength compared to their coarse-grained counterparts because of the high density of grain boundary interfaces (Van Swygenhoven, 2002). At sub-micron grain sizes, the grain boundaries act as barriers to the motion of dislocations, which result in forest hardening (Kacher et al., 2014). It has been well-established that decreasing the grain size significantly increases the flow stress of the polycrystalline metal, following the Hall-Petch relationship, due to the increased density of grain boundaries. However, various deviations from the Hall-Petch equations have been reported at grain sizes of several tens of nanometers (Schiotz et al., 1998; Schiotz and Jacobsen, 2003; Lu et al. 2009; Li et al., 2010). This change in deformation response has been attributed to the significantly lower density of preexisting dislocations within the individual grains, as well as nanoscale confinement effects which severely limit the operation of traditional dislocation sources, such as Frank-Read sources. At these length-scales, the grain boundaries instead become directly involved in the accommodation of strain and serve as active sources for dislocation emission (Yamakov et al., 2002; Shan et al., 2004; Li et al., 2010). Other possible grain boundary-mediated processes that can be activated include grain boundary sliding, grain boundary migration and grain rotation (Sansoz and Molinari 2004, 2005; Warner et al., 2006; Cahn et al., 2006; Zhang et al., 2005; Yamakov et al., 2004). Understanding and quantifying the relationship between these interface mechanisms and the grain boundary structure, through the development of grain boundary descriptors, is a step towards the a priori engineering of grain boundaries.

Fundamentally, the deformation of crystalline metals occurs by the collective slip of adjacent close-packed planes of atoms under the action of stress (Hull and Bacon, 2001). The initiation of slip takes place when the resolved shear stress, $\tau$, along the slip direction exceeds a threshold 
value known as the critical resolved shear stress $\tau_{0}$. While $\tau$ is greatly affected by the orientation of the slip plane and slip direction with respect to the loading axis per Schmid's law, slip will be equally favored under applied external tension or compression albeit in opposite directions. However, molecular dynamics (MD) simulations on perfect single crystals with no initial dislocation content reported an asymmetry in the stress required for homogeneous partial dislocation nucleation under applied uniaxial tensile and compressive loads (Tschopp and McDowell 2007a; Xie et al., 2014). In addition, MD simulations on symmetrical-tilt grain boundaries demonstrate that certain grain boundary orientations require a higher dislocation nucleation stress under uniaxial compression than tension, and vice-versa for other grain boundary orientations (Tschopp et al., 2008; Shimokawa, 2010; Uranagase and Matsumoto, 2016). This tension-compression asymmetry was attributed to: (a) the increased $\tau_{0}$ associated with compressive stress acting normal to the slip plane resulting in a higher yield strength in compression than in tension (Tschopp et al., 2008), as well as (b) the varying internal structure of the grain boundaries, which may favor slip in one direction over another (Shimokawa, 2010).

A key obstacle to understanding the tension-compression asymmetrical response of grain boundaries is the lack of suitable quantitative interface descriptors. The grain boundary energy, for example, quantifies the excess free energy of the interface. Grain boundaries with low energy tend to have good atomic fit, but there is no correspondence between this global descriptor and its ability to emit dislocations (Olmsted et al., 2009). A common approach is to divide the local atomic configuration of grain boundaries into distinct structural units (SUs) (Sutton and Vitek, 1983), and to relate the propensity of dislocation emission to the presence of certain unique SUs. For example, symmetrical-tilt grain boundaries containing E-SUs were found to consistently emit dislocations at much lower tensile stress (Tschopp et al., 2007). This unique behavior of E- 
SUs was associated with the high local porosity within the SU which facilitated the early onset of dislocation nucleation. However, no clear correlation has been found between the local porosity of the grain boundary and its critical tensile strength to trigger dislocation emission. Moreover, the porosity of grain boundaries alone cannot explain the surprisingly high nucleation stress for E-SU-containing grain boundaries under uniaxial compression loading, in contrast to its ductile tensile response.

Recently, we have reconstructed the equivalent traction fields along $\langle 110\rangle$ symmetrical-tilt $\mathrm{Ni}$ grain boundaries from local atomic stress information in the vicinity of the equilibrium grain boundary structures using field projection methods ( $\mathrm{Li}$ and Chew, 2016). These equivalent grain boundary tractions were successfully used to predict the critical stress required to trigger the emission of dislocations from all E-SU-containing grain boundaries under applied tension loading. Here, we adopt these traction-based descriptors to quantify the tension-compression asymmetrical dislocation nucleation processes from a wide range of $\langle 110\rangle$ symmetrical-tilt $\mathrm{Ni}$ grain boundaries. We show that the resolved shear stress contribution from the grain boundary tractions either assists or prevents the emission of Shockley partial dislocations, depending on its direction with respect to the resolved shear stress contribution from external tensile or compressive loading. Our predictions of the critical emission stress from the grain boundary traction signatures under applied tension or compression loading, are in perfect agreement with postdictive MD simulation results for readily-emitted Shockley partials along the boundary. Our results also clarify the atomistic mechanisms underlying the emission of extrinsic stacking faults and non-Schmid dislocations from certain grain boundary orientations subjected to external compression.

\section{Molecular Dynamic Simulations}


We study the asymmetrical dislocation emission processes of $\langle 110\rangle$ symmetrical-tilt Ni grain boundaries under applied uniaxial tension or compression with molecular dynamics (MD) simulations. Our MD simulations are performed using LAMMPS (Plimpton, 1995). The interatomic interaction between atoms is governed by the embedded atom method (EAM) potential for Ni (Adams et al., 1989). This potential has been shown to closely reproduce the $\mathrm{C}_{11}$, $\mathrm{C}_{12}$, and $\mathrm{C}_{44}$ elastic constants of $\mathrm{Ni}$, and consequently the bulk and shear modulus (Rassoulinejad-Mousavi et al., 2016). In addition, our calculated critical resolved shear strength of $\tau_{0} \sim 5.1 \mathrm{GPa}$ for $\{111\}<112>$ partial dislocation slip based on this potential is in perfect agreement with the $\sim 5 \mathrm{GPa}$ ideal shear strength from density functional theory (DFT) calculations (Shang et al., 2012). We focus on grain boundary structures with tilt angles of $\theta<50^{\circ}$, and $\theta>120^{\circ}$. Our MD simulations demonstrate that grain boundary structures with $55^{\circ}<\theta<100^{\circ}$ undergo grain boundary migration and grain rotation under applied uniaxial tension or compression, the mechanisms of which have been previously studied for the case of the $\Sigma 3(112)$ incoherent twin boundary (Wang et al., 2011b). Each bilayer grain boundary model structure is constructed by first rotating two perfect face-centered-cubic (FCC) Ni crystals by $\pm \frac{\theta}{2}$ about the [110] $\left(x_{3}\right)$ axis, with the grain boundary located along the $x_{1}$ axis. For each $\theta$, we create multiple possible grain boundary configurations by laterally displacing the top crystal with respect to the bottom crystal in $1 \%$ increments of the unit structural length along the $x_{1}$ direction; the overlapping atoms are deleted, and the resulting grain boundary configurations are subjected to energy minimization (Tschopp and McDowell, 2007b; Cheng et al., 2010). Then, we select the configuration with the lowest grain boundary energy for each $\theta$ as the representative grain boundary structure. The grain boundary energy of these structures are in near-perfect agreement with previous studies (Olmsted et al., 2009; Sangid et al., 2010). Where possible, we have 
validated the atomic configurations of these grain boundary structures with that obtained from transmission electron microscopy (TEM) imaging or from prior atomic simulations (Rittner and Seidman, 1996; Krakow et al., 1986; Priester, 2001; Sansoz and Molinari, 2005). Our bilayer grain boundary structures are periodic along all three directions, and have $\left(x_{1}, x_{2}, x_{3}\right)$ model dimensions of $\sim 10 \times 20 \times 10 \mathrm{~nm}^{3}$. We subject these model structures to an NVT ensemble, with temperature maintained at $500 \mathrm{~K}$ with a Nose-Hoover thermostat for $0.05 \mathrm{~ns}$. Using an NPT ensemble, we subsequently quench these structures to the target temperature of $10 \mathrm{~K}$ within 0.03 ns, while keeping the simulation box to be pressure-free in all three directions with a Berendsen barostat. All our MD simulations are performed with a fixed time step of 1 fs. The resulting grain boundary structures are composed of repeated structure units (SUs) with periodic length along the $x_{2}$ direction, $d_{p}$, as summarized in Fig. 1 for $\theta<50^{\circ}$ and Fig. 2 for $\theta>120^{\circ}$.

Using the model structures above, we perform uniaxial tension (compression) simulations by stretching (shrinking) the simulation box along the $x_{2}$ direction, i.e. normal to the grain boundary, at a strain rate of $10^{8} / \mathrm{s}$, while maintaining stress-free boundary conditions in the $x_{1}$ and $x_{3}$ directions with a Nose-Hoover barostat. The system is equilibrated to the target temperature of $10 \mathrm{~K}$ throughout the deformation process. Fig. 3 shows the absolute tensile and compressive stress-strain response of our grain boundary structures. We calculate the macroscopic $\left|\sigma_{22}\right|$ stress by averaging the virial stress component of all the atoms over the deformed simulation box volume (Cheung and Yip, 1991). For each grain boundary structure, the abrupt loss of stresscarrying capacity is associated with the first nucleation of dislocations in the model structure, and the peak $\left|\sigma_{22}\right|$ stress is termed as the critical tensile or compressive strength for dislocation emission. We consistently observe tension-compression asymmetry in the critical strength for emission of dislocations across all ten <110> symmetrical-tilt grain boundary structures 
considered here. Grain boundaries with $\theta<50^{\circ}$ (Fig. 3a) exhibit higher critical strength of $\sim 12$ $14 \mathrm{GPa}$ under tension, as compared to the $\sim 5 \mathrm{GPa}$ critical strength under compression. In contrast, grain boundaries with $\theta>120^{\circ}$ (Fig. 3b) display critical high strengths of $15-25 \mathrm{GPa}$ under compression, but readily emit dislocations under tension with much lower critical strengths of $\sim 3-5 \mathrm{GPa}$.

Fig. 4 shows the deformed atomic configurations at the first instant of dislocation emissions for four representative grain boundaries under uniaxial tension and compression loading. The grain boundary structures are filtered based on the centro-symmetric parameter to display only non-FCC atoms, with dislocations colored in red and stacking faults colored in green (Kelchner et al., 1998). Observe that uniaxial compression of the $\Sigma 19(116)$ and $\Sigma 27(115)$ grain boundaries results in the ready emission of edge-type $\frac{1}{6}\langle 112\rangle$ Shockley partials $\left(b_{1}\right)$ from the boundaries. Under tension, however, nucleation of dislocations occurs only in the bulk and at much higher stress levels. Similar observations are made for the remaining $\theta<50^{\circ}$ grain boundary structures

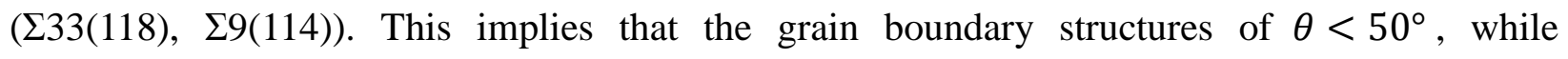
seemingly ductile under compression, have even higher tensile strength than their bulk FCC crystals of the same orientation. In contrast, Shockley partials are readily emitted from preexisting dislocations or E-SUs located along $\theta>120^{\circ}$ grain boundaries under tension (e.g. $\Sigma 33(554)$ and $\Sigma 33(441)$ in Fig. 4). Under compression, however, these grain boundaries can sustain very high loads exceeding $\sim 20 \mathrm{GPa}$ (Fig. 3b), which causes the collapse of the porous ESUs along the boundaries to reform distorted A-SUs. Continued compressive deformation eventually results in the emission of non-Schmid edge-type (001)[1110] full dislocations for $\Sigma 33(554)$ and $\Sigma 11(332)$ grain boundaries, while extrinsic $\{111\}$ stacking faults of a few atomic 
layers thick are formed for the remaining $\theta>120^{\circ}$ grain boundaries $(\Sigma 9(221), \Sigma 27(552)$, $\Sigma 19(331), \Sigma 33(441))$.

\section{Continuum-Equivalent Grain Boundary Tractions}

A number of continuum-based metrics have been established for evaluating the local environments surrounding atoms. Stukowski (2012) proposed neighbor distance analysis to identify atomic SUs in grain boundaries. Zimmerman et al. (2009) and Tucker et al. (2010) derived expressions for calculating the atomic-scale deformation gradients within atomistic simulations which were consistent with continuum mechanical concepts. Zimmerman et al. (2010) and Zhang et al. (2015) also presented methods to extract continuum mechanical variables such as stress from MD simulations.

In order to represent the atomic structure of a grain boundary quantitatively, we consider a plane of cut across an equilibrium grain boundary structure. A distribution of shear and normal tractions has to be introduced along this plane of cut to maintain the same disruption in the atomic arrangement caused by the presence of the grain boundary, and hence the local stress field. These traction distributions are then taken to represent the local grain boundary structure. Here, we inversely reconstruct the grain boundary traction distributions from local atomic stress information in the vicinity of the boundary using field projection methods (Chew et al., 2009; Kim et al., 2012; Chew, 2013; Chew, 2014).

Fig. 5a shows a schematic of a domain $V$ of width $L$ and height $H$ bounded by surface $S$ (red solid and dashed lines) on the upper grain, with the grain boundary centered along the dashed lines. From the principle of virtual work for a static system without body force, the traction $t_{i}$ 
along the boundary $S$ is related to the Cauchy stress $\sigma_{i j}$ within the domain $V$ by (Wang et al., 2011a; Li and Chew, 2016):

$$
\int_{V} \delta \boldsymbol{\varepsilon}: \boldsymbol{\sigma} \mathrm{d} v=\int_{S} \delta \boldsymbol{u} \cdot \boldsymbol{t} \mathrm{d} s=\Delta \Phi
$$

where $\delta u_{i}$ is the admissible virtual displacement field, $\delta \varepsilon_{i j}$ is the associated virtual strain field, and $\Delta \Phi$ represents the variation in the total interatomic potential energy. The shear and normal traction distributions along the red dashed lines are represented in terms of a Fourier series expansion:

$$
t_{i}\left(x_{1}\right)=\sum_{k=1}^{n} A_{k}^{i} \sin \frac{2 k \pi x_{1}}{L}+\sum_{k=1}^{n} B_{k}^{i} \cos \frac{2 k \pi x_{1}}{L}
$$

where $k$ is the wave number, $n$ is the number of Fourier terms, and $A_{k}^{i}, B_{k}^{i}$ are the Fourier coefficients to be determined with $i=1,2$. To calculate these two groups of Fourier coefficients, we define two continuous and smooth virtual displacement fields:

$$
\begin{aligned}
\delta u_{i}^{1}\left(x_{1}, x_{2}\right) & =\epsilon F\left(x_{1}, x_{2}\right) \sin \frac{2 k \pi x_{1}}{L} \\
\text { and } \quad \delta u_{i}^{2}\left(x_{1}, x_{2}\right) & =\epsilon F\left(x_{1}, x_{2}\right) \cos \frac{2 k \pi x_{1}}{L}
\end{aligned}
$$

where $\epsilon=0.01$ is the amplitude of the displacement variation, and $F\left(x_{1}, x_{2}\right)$ is a perturbation envelope function:

$$
F\left(x_{1}, x_{2}\right)=\left\{\begin{array}{cr}
0 & \left(x_{1}, x_{2}\right) \in D_{0} \\
\left(\frac{H}{x_{2}}-1\right)\left(1+\frac{2 x_{1}}{L}\right) & \left(x_{1}, x_{2}\right) \in D_{1} \\
1-\frac{x_{2}}{H} & \text { for } \quad\left(x_{1}, x_{2}\right) \in D_{2} \\
\left(\frac{H}{x_{2}}-1\right)\left(1-\frac{2 x_{1}}{L}\right) & \left(x_{1}, x_{2}\right) \in D_{3} \\
1 & \left(x_{1}, x_{2}\right) \in D_{4}
\end{array}\right.
$$


designed to achieve $\delta \boldsymbol{u} \cdot \boldsymbol{t}=0$ along the solid red lines and $\delta \boldsymbol{u} \cdot \boldsymbol{t} \neq 0$ along the dashed red line representing the interface of interest (grain boundary) in Fig. 5a.

In MD simulations, the virial definition of stress is often interpreted as the Cauchy equivalent stress. Similarly, we use the local atomic stress information from MD to represent the average Cauchy equivalent stress $\boldsymbol{\sigma}$ within the Voronoi cell around each atom (Zimmerman et al., 2004; Subramaniyan and Sun, 2008). Substituting (2) to (4) in (1), the unknown Fourier coefficients can be expressed as $A_{k}^{i}=\frac{2 \Delta \Phi_{k}^{1}}{\epsilon L}, B_{k}^{i}=\frac{2 \Delta \Phi_{k}^{2}}{\epsilon L}$, from which we obtain $t_{i}\left(x_{1}\right)$. We have previously shown that both the shear $\left(t_{1}\right)$ and normal $\left(t_{2}\right)$ traction distributions rapidly converge with increasing Fourier terms, and are generally in good agreement with discrete virial stresses of atoms close to the boundary interface of interest ( $\mathrm{Li}$ and Chew, 2016). The reconstructed grain boundary tractions satisfy both compatibility and equilibrium requirements, and are therefore continuum-equivalent descriptors for the grain boundary atomic structures.

Grain boundary structures are typically of finite thickness, and the extracted traction distributions will be sensitive to the plane of cut (dashed red line in Fig. 5a) taken along the finite thickness dimension $\left(x_{2}\right)$. To accurately predict the critical stress for emission of dislocations from the boundary, the traction distributions should be centered about the cores of pre-existing dislocations along the boundary or at locations where slip first initiates. These dislocation nucleation sites typically have high resolved shear stress along the active slip-plane ( $\mathrm{Li}$ and Chew, 2016). Fig. 5b shows the equilibrium $\Sigma 27(115)$ grain boundary structure. We consider three planar cuts (red, blue and magenta) taken about the finite grain boundary thickness in Fig. $5 \mathrm{~b}$, and show the corresponding shear $\left(t_{1}\right)$ and normal $\left(t_{2}\right)$ traction distributions in Fig. $5 \mathrm{c}$ and $5 \mathrm{~d}$ respectively. The $t_{1}$ tractions are negligible along the center of the boundary (red line) due to the 
symmetrical structure of $\Sigma 27(115)$. The $t_{2}$ tractions, however, fluctuate between positive and negative peaks of $\sim \pm 30 \mathrm{GPa}$ within a single atomic spacing at periodic sites along the boundary. At these sites, the equal but opposite acting tractions on pairs of neighboring atoms contribute to shear dominant loading along the two neighboring closed-packed $\{111\}$ slip planes in the <112> slip direction, as denoted by white arrows in Fig. 5b for slip in the upper grain. Hence, the sharp transition in the $t_{2}$ tractions between atom pairs on neighboring slip planes are indicative of initiation sites for dislocation slip. This has been confirmed by postdictive MD simulations under uniaxial compression, as shown by the deformed atomic configuration of the $\Sigma 27(115)$ grain boundary in the inset in Fig. 5b at the instant of Shockley partial emission. The fluctuations in the $t_{2}$ traction peaks decay with increasing $x_{2}$ distance from the initiation sites for dislocation slip, due to the decay of the local stress field. The $t_{1}$ tractions become more distinctive some distance away (blue line) since the structure is no longer symmetric, but rapidly decreases to 0 once the plane-of-cut (magenta line) is sufficiently far away from the initiation sites for slip.

Fig. 6 summarizes the traction signatures taken along yellow dashed lines of the equilibrium $\Sigma 19(116), \Sigma 33(554)$, and $\Sigma 33(441)$ grain boundary structures. Our simulations show that Shockley partials are readily emitted from $\theta<50^{\circ}$ grain boundaries (represented by $\Sigma 19(116)$, and $\Sigma 27(115)$ in Fig. 5b) only under compression, but are readily emitted from $\theta>120^{\circ}$ grain boundaries (represented by $\Sigma 33(554)$ and $\Sigma 33(441)$ ) only under tension. In both cases, the transition in the $t_{2}$ traction peaks between neighboring atom pairs from positive to negative or vice-versa, assisted at times by similar transition in the $t_{1}$ traction peaks (in the case of $\Sigma 33(554))$, results in high resolved shear stresses along the $\{111\}<112>$ slip system. The deformed configurations of all three grain boundaries at the instant of Shockley partial emission in the inset confirms that slip indeed initiates from these traction transition sites. The traction 
transition sites for all $\theta<50^{\circ}$ and $\theta>120^{\circ}$ grain boundaries are denoted by yellow and green open circles in Figs. 1 and 2 for the ready emission of Shockley partials into the upper and lower grains.

\section{Results}

\subsection{Traction-assisted Shockley partial emission}

The low critical strength of $\theta<50^{\circ}$ grain boundaries under uniaxial compression and $\theta>120^{\circ}$ grain boundaries under uniaxial tension is associated with the ready emission of edgetype $<112>\{111\}$ Shockley partials $\left(\boldsymbol{b}_{1}\right)$. The traction signatures of the equilibrium atomic configurations for these grain boundaries (Figs. 5b and 6) demonstrate high resolved shear stress $\tau_{\mathrm{GB}}$ along the active $<112>\{111\}$ slip-systems prior to loading, which assists one plane of atoms to slide more readily over its neighboring plane of atoms. To calculate $\tau_{\mathrm{GB}}$, we adopt a finite element half-space model comprising of a parallelogram of length $d_{p}$ in the $x_{1}$ direction, representing the upper grain of a single grain boundary period, which is inclined at an angle $\alpha$ denoting the angle between grain boundary interface and the possible (111) slip planes for Shockley partial emissions (Fig. 7a). The inclined boundaries on the left and right edges of the finite element model are tied together to simulate periodic boundary conditions, and the continuum-equivalent $t_{1}$ and $t_{2}$ grain boundary tractions are applied to the lower boundary of the half-space model. We adopt 4-noded bilinear plane strain elements to model the upper grain, and introduce infinite elements at the upper boundary to represent the bulk crystal. The material properties are elastic anisotropic, with elastic constants $c_{11}=247 \mathrm{GPa}, c_{44}=125 \mathrm{GPa}$ and $c_{12}$ $=148 \mathrm{GPa}$ defined in the three <100> material orientations (Adams et al., 1989). To model initial slip along the equilibrium grain boundaries solely associated with the grain boundary tractions, 
we introduce user-defined 4-noded cohesive zone elements along the $\{111\}<112>$ possible emission pathways (dashed lines in Fig. 7a), which are pre-determined based on the traction signatures of the respective grain boundaries. The shear traction versus separation relationship for partial dislocation slip (solid black curve in Fig. 7b) is calculated in MD by taking the gradient of the generalized stacking fault energy (GSFE) surface of (111) Ni along the <112> direction. We fit this MD-derived traction-separation relationship to an exponential tractionseparation law (dashed curve in Fig. 7b) (Xu and Needleman, 1994), which governs the relative shear tractions versus shear displacements of the cohesive elements along the $\langle 112\rangle$ slip plane. Our finite element calculations are performed using the commercial finite element code Abaqus 6.13.

The above finite element model is then used to compute the grain boundary slidingseparation along the slip-plane, and in turn the resolved shear stress contribution, $\tau_{\mathrm{GB}}$, from the traction distributions of the equilibrium (undeform) grain boundary structure. To nucleate a Shockley partial from the boundary, the total resolved shear stress contributions from both $\tau_{\mathrm{GB}}$ and external loading $\tau_{\text {ext }}=m \sigma_{22}$ must exceed the critical barrier stress of $\tau_{0} \sim 5.1 \mathrm{GPa}$ for shear-slip (Fig. 7b), where $\sigma_{22}$ is the applied stress, and $m$ is the Schmid factor of the slip system associated with Shockley partial emission from the boundary. Hence, the critical tensile or compressive strength of the grain boundary, associated with the ready emission of Shockley partials, can be derived as

$$
\sigma_{22}^{c}=\left(\tau_{0}-\tau_{\mathrm{GB}}\right) / m
$$

As shown schematically in Fig. 7c for external tensile and compressive loading, however, $\tau_{\mathrm{GB}}$ only assists sliding of the slip-planes if $\tau_{\text {ext }}$ acts in the same direction as $\tau_{\mathrm{GB}}$. If $\tau_{\mathrm{GB}}$ acts in the 
opposite direction of $\tau_{\text {ext }}$, then, $\tau_{\text {ext }}$ must overcome both $\tau_{0}$ and $\tau_{\mathrm{GB}}$ to allow Shockley partial emission based on (5); the actual $\tau_{\text {ext }}$ required could be even higher in practice, since the emission of Shockley partials (e.g. $\frac{1}{6}[\overline{1} 1 \overline{2}]$ ) in the reverse direction $([1 \overline{1} 2])$ requires the concurrent slip of two $\left(\frac{1}{6}[211]\right.$ and $\left.\frac{1}{6}[\overline{1} \overline{2} 1]\right)$ Shockley partials. This explains why grain boundary structures which readily emit dislocations under compression $\left(\theta<50^{\circ}\right)$ tend to display high nucleation strength under tension, while those which readily emit dislocations under tension $\left(\theta>120^{\circ}\right)$ display high nucleation strength under compression. Our traction signatures further confirm that $\tau_{\mathrm{GB}}$ for grain boundary structures with $\theta<50^{\circ}$ acts in the direction of $\tau_{\text {ext }}$ from uniaxial compression (Fig. 7c, lower), while $\tau_{\mathrm{GB}}$ for grain boundary structures with $\theta>120^{\circ}$ acts in the direction of $\tau_{\text {ext }}$ from external tension (Fig. 7c, upper). Our predictions of $\sigma_{22}^{c}$ based on (5) for the ready emission of Shockley partial dislocations, as summarized in Fig. 8, are in perfect agreement with postdictive MD simulation results.

\subsection{Traction-impeded dislocation emission}

When $\tau_{\text {ext }}$ generated by external loading acts in the opposite direction of $\tau_{\mathrm{GB}}$, the suppression of Shockley partial emission by the grain boundary tractions infers that other dislocation mechanisms could instead be activated. For the $\theta<50^{\circ}$ grain boundaries, dislocations nucleate from the bulk instead of from the boundaries (see $\Sigma 19(116)$ and $\Sigma 27(115)$ in Fig. 4). Hence, the $\sim 12 \mathrm{GPa}$ critical tensile strength for the $\theta<50^{\circ}$ grain boundaries are almost the same as that in perfect FCC crystals with the same tilt orientations ( $\mathrm{Li}$ and Chew, 2016). For the $\theta>120^{\circ}$ grain boundaries which favor the ready emission of dislocations only under tension, the high sustainable compressive stress of these boundaries introduces two new dislocation mechanisms: emission of non-Schmid full dislocations for $\Sigma 33(554)$ and $\Sigma 11(332)$ grain boundaries, and 
formation of extrinsic stacking faults for $\Sigma 9(221), \Sigma 27(552), \Sigma 19(331)$ and $\Sigma 33(441)$ grain boundaries.

Non-Schmid full dislocation emission

During compressive deformation of the $\Sigma 33(554)$ grain boundary, pre-existing dislocations $\left(b_{1}\right)$ along the boundary (Fig. 2) first reform E-SUs. Under further compression, each of these porous E-SUs in turn collapses to form two distorted A-SUs, as shown by the deformed atomic configuration prior to dislocation emissions in Fig. 9a. This reformation and subsequent collapse of the E-SUs along the boundary is completed under large compressive loads of $\sim 15 \mathrm{GPa}$, since the resolved shear stress generated by external loads must overcome those from pre-existing grain boundary tractions. Beyond this point, non-Schmid (001)[110] full dislocations, rather than Shockley partials, are emitted from the boundary, as shown in Fig. 9b. To understand the mechanisms underlying this process, we project in Fig. 9c the traction distributions along the deformed $\Sigma 33(554)$ grain boundary structure corresponding to Fig. 9a. Here, $\hat{t}_{1}$ and $\hat{t}_{2}$ represent the uniform (mean) shear and normal tractions introduced by external compression, while the plotted $\left(t_{1}-\hat{t}_{1}\right)$ and $\left(t_{2}-\hat{t}_{2}\right)$ distributions represent the fluctuations in shear and normal tractions caused by the non-uniform arrangement of atoms along the deformed grain boundary. We observe similar traction profiles compared to that along the undeformed $\Sigma 33(554)$ grain boundary structure in Fig. 6. However, the reconfigured atomic structure of the grain boundary now exhibits two-fold higher compression-tension peaks for $\left(t_{2}-\hat{t}_{2}\right)$. In addition, the compression-tension peaks are now shifted to atom pairs along neighboring (001) planes; the high $\tau_{\mathrm{GB}}$ along the (001) [110] slip system, as denoted by arrows in Fig. 9b, then results in the emission of full dislocations. This is in contrast to the deformation process of $\Sigma 33(554)$ under 
tension, where fluctuations in the traction signatures are centered about atom pairs along neighboring (111) planes.

The critical external load, $\sigma_{22}^{c}$, to trigger the emission of (001) [110] full dislocations from the $\Sigma 33(554)$ grain boundary under compression can be ascertained from (5). We compute $\tau_{\mathrm{GB}}$ of the deformed grain boundary atomic configuration in Fig. 9a from our finite element half-space model with imposed $\left(t_{1}-\hat{t}_{1}\right)$ and $\left(t_{2}-\hat{t}_{2}\right)$ traction distributions along the lower boundary. The cohesive elements in this new finite element model are now along the [110] direction, and the associated traction-separation law is calculated from the gradient of the GSFE along the $\{001\}$ plane in the $\langle 110\rangle$ direction. Because of the high external compressive stress of $\sigma_{22}^{\text {ext }}=-18.7$ GPa just prior to initiation of slip, the $\{001\}$ plane of the crystal structure experiences high normal stress of $\sigma_{n}=\sigma_{22}^{e x t} \cos ^{2} \alpha$, where $\alpha$ denotes the angle between the grain boundary interface and the relevant $\{001\}$ slip plane. This high compressive normal stress will significantly increase the GSFE along the $\{001\}$ plane (Tschopp et al., 2008). As such, we obtain the GSFE of the $\{001\}$ plane by rigidly displacing the (001)-oriented grains along the $<110>$ direction in MD, while simultaneously imposing $\sigma_{n}$ on the (001) surface. The extracted cohesive zone laws are shown in Fig. 9d (solid curve). Note that neglecting the normal load contribution (dashed curve) underestimates the critical barrier stress $\tau_{0}$ for shear slip (16 GPa versus 18.5 GPa). Our predicted critical strength of $\sigma_{22}^{c}=-20.2 \mathrm{GPa}$ obtained from (5) is in close agreement with the MD-simulated value of $-18.7 \mathrm{GPa}$. We have performed similar calculations for the $\Sigma 11(332)$ grain boundary, and have obtained critical compressive strength values of $\sigma_{22}^{c}=-20.5 \mathrm{GPa}$, which again is in good agreement with MD-simulated value of $-20.2 \mathrm{GPa}$. 
The formation of extrinsic stacking faults are observed for grain boundaries with tilt angles of $\theta>140^{\circ}(\Sigma 9(221), \Sigma 27(552), \Sigma 19(331)$, and $\Sigma 33(441))$ subjected to uniaxial compression. Similar to the $\Sigma 33(554)$ and $\Sigma 11(332)$ grain boundaries discussed above, the compressive deformation process first collapses the porous E-SUs of these boundaries into two distorted ASUs, as shown for the $\Sigma 33(441)$ grain boundary in Fig. 10a-i. Subsequent deformation induces the emission of a mixed partial $\left(\boldsymbol{b}_{3}=\frac{1}{6}[211]\right)$ along a (111) close-packed plane (Fig. 10a-ii), which is quickly followed by the emission of a second mixed partial $\left(\boldsymbol{b}_{2}=\frac{1}{6}[\overline{1} \overline{2} 1]\right)$ along another parallel (111) close-packed plane two (111)atomic planes away (Fig. 10a-iii). The emission of this second $b_{2}$ partial cancels out any out-of-plane (110) deformation, i.e.

$$
\frac{1}{6}[211]+\frac{1}{6}[\overline{1} \overline{2} 1]=\frac{1}{6}[1 \overline{1} 2]\left(-\boldsymbol{b}_{1}\right)
$$

Hence, the crystal structure is displaced only along the [1 $1 \overline{1} 2]$ direction, leaving behind a single atomic-layer thick extrinsic $\{111\}$ stacking fault. A top view of the (1111) closed-packed planes during the extrinsic stacking fault formation process, with schematic illustration, is shown in Fig. 10b: grey atoms denote the $\{111\}$ stacking fault plane (B) with neighboring $\{111\}$ planes represented by yellow (A) and green atoms (C). The grey and green planes, as well as the crystal structure below, first slips in the [211] direction with the emission of a $\boldsymbol{b}_{3}$ partial (compare Fig. 10b-i and Fig. 10b-ii), which is followed by slip of the green plane and crystal structure below in

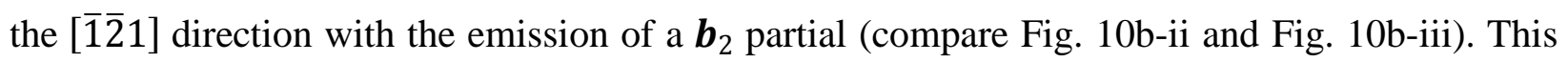
process leaves behind the extrinsic stacking fault, represented by the grey plane. As observed in our MD simulations, the formation of extrinsic stacking fault can also occur by the emission of $\boldsymbol{b}_{2}$ partials prior to $\boldsymbol{b}_{3}$ partials, depending on the local out-of-plane $\left(x_{3}\right)$ stress state along the grain boundary. Similar mechanisms are also displayed by the $\Sigma 9(221), \Sigma 27(552)$ and $\Sigma 19(331)$ 
grain boundaries under uniaxial compression, albeit with different extrinsic stacking fault thicknesses. We remark that this deformation mechanism is strikingly different from that exhibited by similarly-oriented $\langle 110\rangle \mathrm{Cu}$ grain boundaries, where simultaneous emissions of partial dislocations from the boundary resulting in the formation of hexagonal-closed-packed (HCP) phase have been reported (Tschopp et al., 2008).

The reconfiguration of the grain boundary structure brought about by the collapse of the ESUs into two A-SUs leads to a reconfiguration of the local stress field near the boundary. Comparison of the traction signatures of the equilibrium $\Sigma 33(441)$ grain boundary (Fig. 6) versus the deformed grain boundary just prior to emission of $\boldsymbol{b}_{3}$ partials (Fig. 10a-i) shows that the maximum fluctuations in the traction peaks, and consequently the initiation sites for slip, have now shifted. However, these fluctuations in the traction peaks are still centered about two (111) close-packed planes. Along the favored $(\overline{1} 11)[\overline{1} 1 \overline{2}]$ slip system, $\tau_{\mathrm{GB}}$ generated by these grain boundary tractions is in the same direction as $\tau_{\mathrm{ext}}$, but both $\left(\tau_{\mathrm{GB}}+\tau_{\mathrm{ext}}\right)$ act in opposite direction

to the $\boldsymbol{b}_{1}=\frac{1}{6}[\overline{1} 1 \overline{2}]$ Shockley partial emission direction. Unlike full dislocations, emission of partial dislocations can only happen in one direction (i.e. $+\boldsymbol{b}_{1}$ ) (Xie et al., 2014). In this case, where $\left(\tau_{\mathrm{GB}}+\tau_{\mathrm{ext}}\right)$ act in the reverse direction (i.e. $\left.-\boldsymbol{b}_{1}\right)$, the asymmetry of partial dislocation slip requires the near-simultaneous emission of $\left(\boldsymbol{b}_{2}+\boldsymbol{b}_{3}\right)$ partials to create the extrinsic stacking faults seen in our MD simulations.

\section{Discussions and Conclusion}

The tension-compression asymmetry in the stress required for dislocation nucleation from symmetrical-tilt grain boundaries has previously been attributed to both the role of the resolved stress normal to the slip-planes, and the structure of the grain boundary (Tschopp et al., 2008; 
Shimokawa, 2010). Our results here quantifies how the local atomic structure along the grain boundary, as represented by the traction signatures, ultimately results in the asymmetrical strength of the boundary under tension and compression. The origin of dislocation emission along the boundary, and the resolved shear stress contributions from the disruption in atomic arrangement along boundary, $\tau_{\mathrm{GB}}$, can be quantitatively ascertained from the grain boundary traction signatures. When $\tau_{\mathrm{GB}}$ acts in the direction of the resolved shear stress contribution from external loading, $\tau_{\text {ext }}$, dislocations are readily emitted at lower stresses. When $\tau_{\mathrm{GB}}$ opposes $\tau_{\text {ext }}$, the grain boundaries can then sustain much higher stresses prior to dislocation emission; the larger sustainable loads will infer a larger resolved stress normal to the slip-plane which affects the critical barrier stress $\tau_{0}$ for shear slip. The dependence on the direction of $\tau_{\text {ext }}$ with respect to $\tau_{\mathrm{GB}}$ explains why certain grain boundary orientations $\left(\theta>120^{\circ}\right)$ readily emit dislocations under uniaxial tension but not compression, while others $\left(\theta<50^{\circ}\right)$ instead readily emit dislocations under uniaxial compression but not tension.

The traction signatures along the equilibrium grain boundary structures, and the associated dislocation nucleation sites with high $\tau_{\mathrm{GB}}$, are closely related to the structural units (SUs) along the boundary. The readily-emitted Shockley partials along $\theta>120^{\circ}$ grain boundaries under tension consistently initiate from the E-SUs along the boundary, or in the case of $\Sigma 33(554)$, from pre-existing dislocations dissociated from E-SUs along the boundary. For $\theta<50^{\circ}$ grain boundaries, however, the readily-emitted Shockley partials under compression consistently initiate from pre-existing dislocations or highly-distorted A-SUs along the boundary. The E-SUs are porous regions along the boundary, and the weakened atomic bonding within the E-SUs infers low dislocation nucleation strength under tension loading. In contrast, distorted A-SUs represent high local atom density regions along the boundary. The build-up of stress 
concentration at these high local density sites under compression causes the ready emission of dislocations to relieve the compressive stress; this process transforms the short diagonal of the rhombic A-SU (as viewed in the <110> orientation) to become the long diagonal, and conversely, the long diagonal to become the short one. Compare the A-SUs of the undeform and deformed $\Sigma 27(115)$ grain boundary marked in Fig. 5b. Similar observations have been made in the uniaxial compression of E-SU-containing grain boundaries, where atomic reconfigurations associated with the collapse of the E-SUs under high compressive stress transform each E-SU into two highly-distorted A-SUs (Figs. 9a and 10a-i), from which dislocations are emitted.

The symmetrical-tilt grain boundaries considered in this paper represent the minimum-energy grain boundary state. Because of the short periodic structural length $d_{p}$ of these boundaries, only a small number of SUs can lie within each grain boundary period, as shown in Figs. 1 and 2. This severely limits the number of potential dislocation nucleation sites (A- or E-SUs) within the grain boundary period, each with its own preference for dislocation emission under tension (ESU) or compression (A-SU). Recent studies suggest that actual grain boundaries can occupy a range of metastable configurations due to nonequilibrium processes or simple thermal excitations, resulting in grain boundaries with higher energy states (Han et al., 2016). It is possible for both low-density E-SUs and highly-distorted, denser A-SUs to co-exist along these more realistic grain boundaries, which could allow for the ready emission of dislocations under both tension and compression. This is a subject of future work.

\section{Acknowledgement}

The authors acknowledge the support provided by Dr. Ali Sayir under the AFOSR Aerospace Materials for Extreme Environment Program (award no. FA9550-15-1-0117), as well as 
computational time provided by TACC (award no. TG-MSS130007) and the Blue Waters sustained-petascale computing project which is supported by the National Science Foundation (awards OCI-0725070 and ACI-1238993) and the state of Illinois. Blue Waters is a joint effort of the University of Illinois at Urbana-Champaign and its National Center for Supercomputing Applications.

\section{Reference}

Adams, J. B., Foiles, S. M., \& Wolfer, W. G., 1989. Self-diffusion and impurity diffusion of fcc metals using the five-frequency model and the Embedded Atom Method. J. Mater. Res. 4, 102112.

Cahn, J.W., Mishin, Y., \& Suzuki, A., 2006. Coupling grain boundary motion to shear deformation. Acta Mater. 54, 4953-4975.

Cheng, Y., Jin, Z. H., Zhang, Y. W., \& Gao, H., 2010. On intrinsic brittleness and ductility of intergranular fracture along symmetrical tilt grain boundaries in copper. Acta Mater. 58, 22932299.

Cheung, K. S., \& Yip, S., 1991. Atomic-level stress in an inhomogeneous system. J. Appl. Phys. 70, 5688-5690.

Chew, H. B., Hong, S., \& Kim, K. S., 2009. Cohesive zone laws for void growth-II. Numerical field projection of elasto-plastic fracture processes with vapor pressure. J. Mech. Phys. Solids 57, 1374-1390.

Chew, H. B., 2013. Inverse extraction of interfacial tractions from elastic and elasto-plastic farfields by nonlinear field projection. J. Mech. Phys. Solids 61, 131-144.

Chew, H. B., 2014. Cohesive zone laws for fatigue crack growth: Numerical field projection of the micromechanical damage process in an elasto-plastic medium. Int. J. Solids Struct. 51, 41764187.

Han, J., Vitek. V., \& Srolovitz, D.J., 2016. Grain boundary metastability and its statistical properties. Acta Mater. 104, 259-273.

Hull, D., \& Bacon, D. J., 2011. Introduction to dislocations, fifth ed. Elsevier.

Kacher, J., Eftink, B. P., Cui, B., \& Robertson, I. M., 2014. Dislocation interactions with grain boundaries. Curr. Opin. Solid. State. Mater. Sci. 18, 227-243. 
Kelchner, C. L., Plimpton, S. J., \& Hamilton, J. C., 1998. Dislocation nucleation and defect structure during surface indentation. Phys. Rev. B 58, 11085.

Kim, H. G., Chew, H. B., \& Kim, K. S., 2012. Inverse extraction of cohesive zone laws by field projection method using numerical auxiliary fields. Int. J. Numer. Meth. Eng. 91, 516-530.

Krakow, W., Wetzel, J. T., \& Smith, D. A., 1986. Observation and interpretation of the atomic structure of a $\Sigma=19 /[110](3 \overline{3} 1)$ tilt boundary in Au thin films. Philos. Mag. A 53, 739-754.

Li, R., \& Chew, H. B., 2016. Grain boundary traction signatures: Quantitative predictors of dislocation emission. Phys. Rev. Lett. 117, 085502.

Li, X., Wei, Y., Lu, L., Lu, K., \& Gao, H., 2010. Dislocation nucleation governed softening and maximum strength in nano-twinned metals. Nature 464, 877-880.

Lu, L., Chen, X., Huang, X., \& Lu, K., 2009. Revealing the maximum strength in nano-twinned copper. Science 323, 607-610.

Olmsted, D. L., Foiles, S. M., \& Holm, E. A., 2009. Survey of computed grain boundary properties in face-centered cubic metals: I. Grain boundary energy. Acta Mater. 57, 3694-3703.

Plimpton, S., 1995. Fast parallel algorithms for short-range molecular dynamics. J. Comput. Phys. 117, 1-19.

Priester, L., 2001. "Dislocation-interface" interaction-stress accommodation processes at interfaces. Mater. Sci. Eng. A 309, 430-439.

Rassoulinejad-Mousavi, S.M., Mao, Y., \& Zhang, Y., 2016. Evaluation of copper, aluminum, and nickel interatomic potentials on predicting the elastic properties. J. Appl. Phys. 119, 244304.

Rittner, J. D., \& Seidman, D. N., 1996. <110> symmetric tilt grain-boundary structures in fcc metals with low stacking-fault energies. Phys. Rev. B 54, 6999.

Sangid, M. D., Sehitoglu, H., Maier, H. J., \& Niendorf, T., 2010. Grain boundary characterization and energetics of superalloys. Mater. Sci. Eng. A 527, 7115-7125.

Sansoz, F., \& Molinari, J.F., 2004. Incidence of atom shuffling on the shear and decohesion behavior of a symmetric tilt grain boundary in copper. Scripta Mater. 50, 1283-1288.

Sansoz, F., \& Molinari, J. F., 2005. Mechanical behavior of $\Sigma$ tilt grain boundaries in nanoscale $\mathrm{Cu}$ and Al: A quasicontinuum study. Acta Mater. 53, 1931-1944.

Schiotz, J., Di Tolla, F.D., \& Jacobsen, K.W., 1998. Softening of nanocrystalline metals at very small grain sizes. Nature 391, 561-563.

Schiotz, J., \& Jacobsen, K.W., 2003. A maximum in the strength of nanocrystalline copper. Science 301, 1357-1359.

Shan, Z., Stach, E. A., Wiezorek, J. M. K., Knapp, J. A., Follstaedt, D. M., \& Mao, S. X., 2004. Grain boundary-mediated plasticity in nanocrystalline nickel. Science 305, 654-657. 
Shang, S.L., Wang, W.Y., Wang, Y., Du, Y., Zhang, J.X., Patel, A.D., \& Liu, Z.K., 2012. Temperature-dependent ideal strength and stacking fault energy of fcc Ni: a first-principles study of shear deformation. J. Phys: Condens. Matter 24, 155402.

Shimokawa, T., 2010. Asymmetric ability of grain boundaries to generate dislocations under tensile or compressive loadings. Phys. Rev. B 82, 174122.

Stukowski, A., 2012. Structure identification methods for atomistic simulations of crystalline materials. Modelling Simul. Mater. Sci. Eng. 20, 045021.

Subramaniyan, A. K., \& Sun, C. T., 2008. Continuum interpretation of virial stress in molecular simulations. Int. J. Solids. Struct. 45, 4340-4346.

Sutton, A. P., \& Vitek, V., 1983. On the structure of tilt grain boundaries in cubic metals I. Symmetrical tilt boundaries. Phil. Trans. R. Soc. A 309, 1-36.

Tschopp, M. A., Tucker, G. J., \& McDowell, D. L., 2007. Structure and free volume of <110> symmetric tilt grain boundaries with the E structural unit. Acta Mater. 55, 3959-3969.

Tschopp, M. A., \& McDowell, D. L., 2007a. Tension-compression asymmetry in homogeneous dislocation nucleation in single crystal copper. Appl. Phys. Lett. 90, 121916.

Tschopp, M. A., \& McDowell, D. L., 2007b. Structures and energies of $\Sigma 3$ asymmetric tilt grain boundaries in copper and aluminum. Philos. Mag. 87, 3147-3173.

Tschopp, M. A., Tucker, G. J., \& McDowell, D. L., 2008. Atomistic simulations of tensioncompression asymmetry in dislocation nucleation for copper grain boundaries. Comput. Mater. Sci. 44, 351-362.

Tucker, G.J., Zimmerman, J.A., \& McDowell, D.L., 2010. Shear deformation kinematics of bicrystalline grain boundaries in atomistic simulations. Modelling Simul. Mater. Sci. Eng. 18, 015002 .

Uranagase, M., \& Matsumoto, R., 2016. Tension-compression asymmetry in uniaxial deformation of a magnesium bicrystal with symmetric tilt grain boundary. Comput. Mater. Sci. $118,124-132$.

Van Swygenhoven, H., 2002. Grain boundaries and dislocations. Science 296, 66-67.

Wang, C. K., Chew, H. B., \& Kim, K. S., 2011a. Nanometer Scale Mechanical Behavior of Grain Boundaries. MRS Proc.1297, 1.

Wang, J., Misra, A., \& Hirth, J. P., 2011b. Shear response of $\Sigma 3\{112\}$ twin boundaries in facecentered-cubic metals. Phys. Rev. B 83, 064106.

Warner, D.H., Sansoz, F., \& Molinari, J.F., 2006. Atomistic based continuum investigation of plastic deformation in nanocrystalline copper. Int. J. Plasticity 22, 754-774.

Xie, H., Yu, T., \& Yin, F., 2014. Tension-compression asymmetry in homogeneous dislocation nucleation stress of single crystals $\mathrm{Cu}, \mathrm{Au}, \mathrm{Ni}$ and $\mathrm{Ni}_{3} \mathrm{Al}$. Mater. Sci. Eng. A 604, 142-147. 
Xu, X. P., \& Needleman, A., 1994. Numerical simulations of fast crack growth in brittle solids. J. Mech. Phys. Solids 42, 1397-1434.

Yamakov, V., Wolf, D., Phillpot, S. R., Mukherjee, A. K., \& Gleiter, H., 2002. Dislocation processes in the deformation of nanocrystalline aluminium by molecular-dynamics simulation. Nat. Mater. 1, 45-49.

Yamakov, V., Wolf, D., Phillpot, S.R., Mukherjee, A.K., \& Gleiter, H., 2004. Deformationmechanism map for nanocrystalline metals by molecular dynamics simulation. Nat. Mater. 3, 4347.

Zhang, H., Mendelev, M.I., Srolovitz, D.J., 2005. Mobility of $\Sigma 5$ tilt grain boundaries: Inclination dependence. Scripta Mater. 52, 1193-1198.

Zhang, L., Jasa, J., Gazonas, G., Jerusalem, A., \& Negahban, M., 2015. Extracting continuumlike deformation and stress from molecular dynamics simulations. Comput. Methods Appl. Mech. Engrg. 283, 1010-1031.

Zimmerman, J.A., Bammann, D.J., \& Gao, H., 2009. Deformation gradients for continuum mechanical analysis of atomistic simulations. Int. J. Solids Struct. 46, 238-253.

Zimmerman, J.A., Jones, R.E., \& Templeton, J.A., 2010. A material frame approach for evaluating continuum variables in atomistic simulations. J. Comp. Phys. 229, 2364-2389.

Zimmerman, J. A., Webb III, E. B., Hoyt, J. J., Jones, R. E., Klein, P. A., \& Bammann, D. J., 2004. Calculation of stress in atomistic simulation. Model. Simul. Mater. Sci. Eng. 12, S319. 


\section{Figure Captions}

Figure 1: Repeated structural units of periodicity $d_{p}$ along symmetrical-tilt $\langle 110\rangle \mathrm{Ni}$ grain boundaries with tilt angles of $\theta<50^{\circ}$. The grain boundary structures are colored by the centrosymmetric parameter, with FCC atoms in red, dislocation cores in white, and stacking faults in blue. Initiation sites for ready-emission of Shockley partials $\left(\boldsymbol{b}_{1}\right)$ into the upper and lower grains under applied uniaxial compression are marked by open yellow and green circles.

Figure 2: Repeated structural units of periodicity $d_{p}$ along symmetrical-tilt $\langle 110\rangle \mathrm{Ni}$ grain boundaries with tilt angles of $\theta>120^{\circ}$. The grain boundary structures are colored by the centrosymmetric parameter, with FCC atoms in red, dislocation cores in white, and stacking faults in blue. Initiation sites for ready-emission of Shockley partials $\left(\boldsymbol{b}_{1}\right)$ into the upper and lower grains under applied uniaxial tension are marked by open yellow and green circles.

Figure 3: Stress-strain relationships for symmetrical-tilt $\langle 110\rangle \mathrm{Ni}$ grain boundaries with tilt angles of $\theta<50^{\circ}$ (a) and $\theta>120^{\circ}$ (b) under uniaxial tension and compression.

Figure 4: Deformed atomic configurations of four typical symmetrical-tilt $\langle 110\rangle \mathrm{Ni}$ grain boundaries at the peak tensile (a) and compressive (b) strength corresponding to the instant of dislocation emission; structures are filtered based on the centro-symmetric parameter to display only non-FCC atoms, with dislocations colored in red and stacking faults colored in green.

Figure 5: (a) Calculation domain of the field projection scheme to reconstruct the normal and shear tractions along the grain boundary (red dashed lines) from atomic stress information in the vicinity (enclosed by red lines). (b) Equilibrium atomic configuration of the $\Sigma 27(115)$ grain boundary; yellow dashed lines denote the active slip planes under uniaxial compression, with the deformed atomic configuration in the inset. (c,d) Field-projected shear $t_{1}$ (c) and normal $t_{2}$ (d) tractions along the corresponding colored dashed lines in (b).

Figure 6: Field-projected shear $\left(t_{1}\right)$ and normal $\left(t_{2}\right)$ tractions along the yellow dashed lines of the equilibrium $\Sigma 19(116), \Sigma 33(554)$, and $\Sigma 33(441)$ grain boundary atomic configurations. Ready emission of dislocations, assisted by grain boundary tractions, initiating at the intersections between yellow and black dashed lines with corresponding deformed atomic configurations in the inset.

Figure 7: (a) Calculation of the resolved shear stress contribution $\tau_{\mathrm{GB}}$ from grain boundary shear $\left(t_{1}\right)$ and normal $\left(t_{2}\right)$ tractions from a finite elment half-space model with cohesive zone law implemented along the active slip-planes. (b) Cohesive zone law for shear-slip along the $<112>\{111\}$ slip system, as calculated from the gradient of the generalized stacking fault energy (GSFE) in MD, and fitted to an exponential model implented in FEM. (c) Illustration showing the ready emssion of dislocations only when $\tau_{\mathrm{GB}}$ acts in the same direction as the resolved shear stress contribution from external loading $\tau_{\text {ext }}$.

Figure 8: Summary of the critical tensile (red symbols) and compressive (blue symbols) strength of symmetrical-tilt <110> Ni grain boundaries from MD, and corresponding predictions (black symbols) from the traction signatures of the equilibrium grain boundary structures. 
Figure 9: (a,b) Deformed atomic configurations of the $\Sigma 33(554)$ grain boundary under uniaxial compression, just prior to (a) and at the instant of (b) full dislocation emission. (c) Traction signatures along the deformed grain boundary structure corresponding to (a). (d) Traction separation laws for non-Schmid $\{001\}<110>$ dislocation slip in the absence of normal stresses to the $\{001\}$ slip-plane (dashed line), and at the stress-state corresponding to (a) (solid line).

Figure 10: (a) Deformed atomic configurations depicting sequence of out-of-plane partial dislocation emissions to form extrinsic stacking faults for $\Sigma 33(441)$ grain boundary under uniaxial compression, with traction signatures of the deformed grain boundary structure in (i) just prior to dislocation emission. (b) Top view and schematic representation of the stacking arrangements of ( $\overline{1} 11)$ planes during the partial dislocation emission processes corresponding to (a). 


\begin{tabular}{c|c|c|c|c} 
Name & Atomic Structure & Tilt Angle & $\begin{array}{c}\text { Periodic } \\
\text { Length } \\
d_{p}(\mathrm{~nm})\end{array}$ & $\begin{array}{c}\text { Structural } \\
\text { Units }\end{array}$ \\
\hline$\Sigma 33(118)$ & & $20.1^{\circ}$ & 2.02 & $2 \mathrm{C}+1 \mathrm{~A}+4 \boldsymbol{b}_{1}$ \\
\hline$\Sigma 19(116)$ & & $26.5^{\circ}$ & 1.53 & $2 \mathrm{C}+4 \boldsymbol{b}_{1}$ \\
\hline $527(115)$ & & & & \\
\hline
\end{tabular}

Figure 1: Repeated structural units of periodicity $d_{p}$ along symmetrical-tilt $<110>\mathrm{Ni}$ grain boundaries with tilt angles of $\theta<50^{\circ}$. The grain boundary structures are colored by the centrosymmetric parameter, with FCC atoms in red, dislocation cores in white, and stacking faults in blue. Initiation sites for ready-emission of Shockley partials $\left(\boldsymbol{b}_{1}\right)$ into the upper and lower grains under applied uniaxial compression are marked by open yellow and green circles. 


\begin{tabular}{|c|c|c|c|c|}
\hline Name & Atomic Structure & Tilt Angle & $\begin{array}{c}\text { Periodic } \\
\text { Length } \\
d_{p}(\mathrm{~nm})\end{array}$ & $\begin{array}{l}\text { Structural } \\
\text { Units }\end{array}$ \\
\hline$\Sigma 33(554)$ & & $121.0^{\circ}$ & 2.02 & $2 C+2 b_{1}+6 D$ \\
\hline$\Sigma 11(332)$ & & $129.5^{\circ}$ & 1.17 & $2 \mathrm{E}+2 \mathrm{~A}$ \\
\hline$\Sigma 9(221)$ & & $141.1^{\circ}$ & 0.75 & $2 \mathrm{E}$ \\
\hline$\Sigma 27(552)$ & & $148.4^{\circ}$ & 1.83 & $4 \mathrm{E}+2 \mathrm{~A}$ \\
\hline$\Sigma 19(331)$ & 2 & $153.5^{\circ}$ & 1.09 & $2 \mathrm{E}+2 \mathrm{~A}$ \\
\hline$\Sigma 33(441)$ & & $160.0^{\circ}$ & 1.43 & $2 \mathrm{E}+4 \mathrm{~A}$ \\
\hline
\end{tabular}

Figure 2: Repeated structural units of periodicity $d_{p}$ along symmetrical-tilt $<110>\mathrm{Ni}$ grain boundaries with tilt angles of $\theta>120^{\circ}$. The grain boundary structures are colored by the centrosymmetric parameter, with FCC atoms in red, dislocation cores in white, and stacking faults in blue. Initiation sites for ready-emission of Shockley partials $\left(\boldsymbol{b}_{1}\right)$ into the upper and lower grains under applied uniaxial tension are marked by open yellow and green circles. 

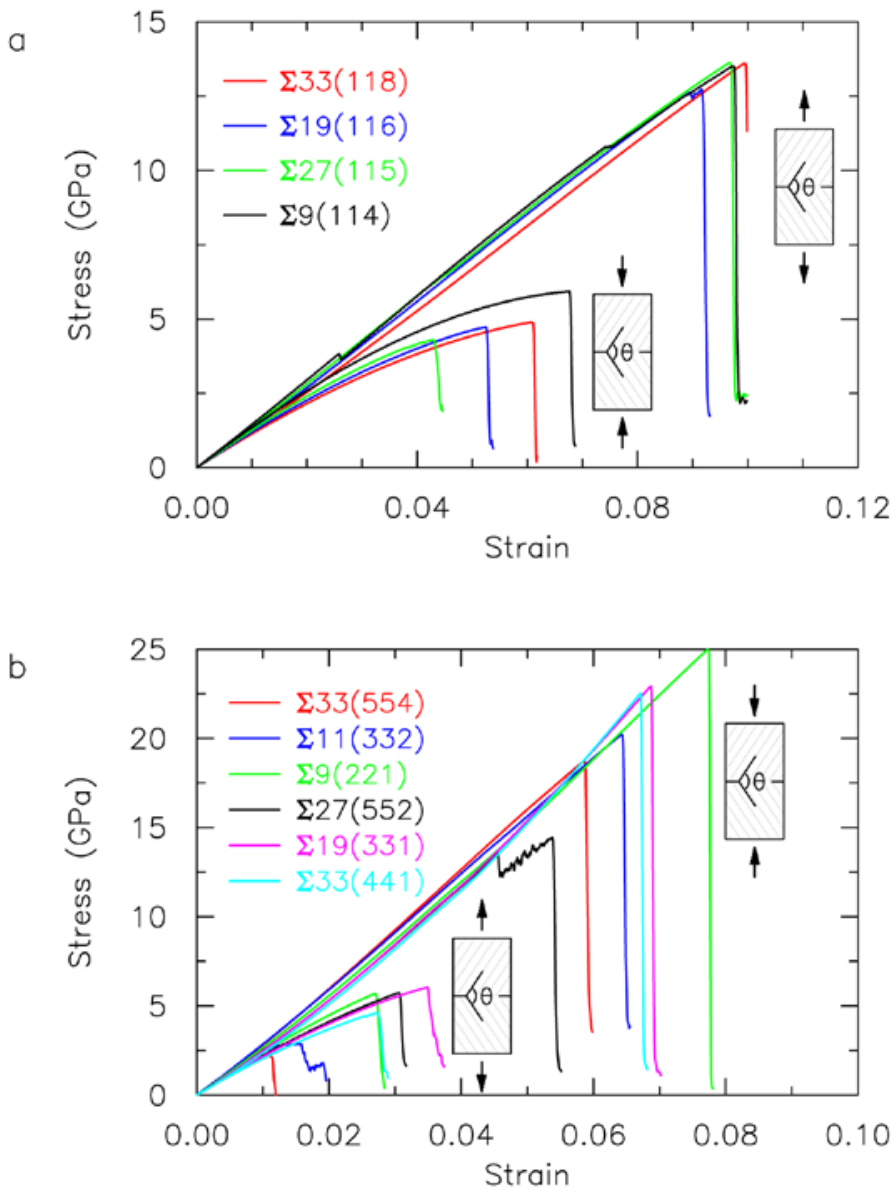

Figure 3: Stress-strain relationships for symmetrical-tilt $<110>\mathrm{Ni}$ grain boundaries with tilt angles of $\theta<50^{\circ}$ (a) and $\theta>120^{\circ}$ (b) under uniaxial tension and compression. 

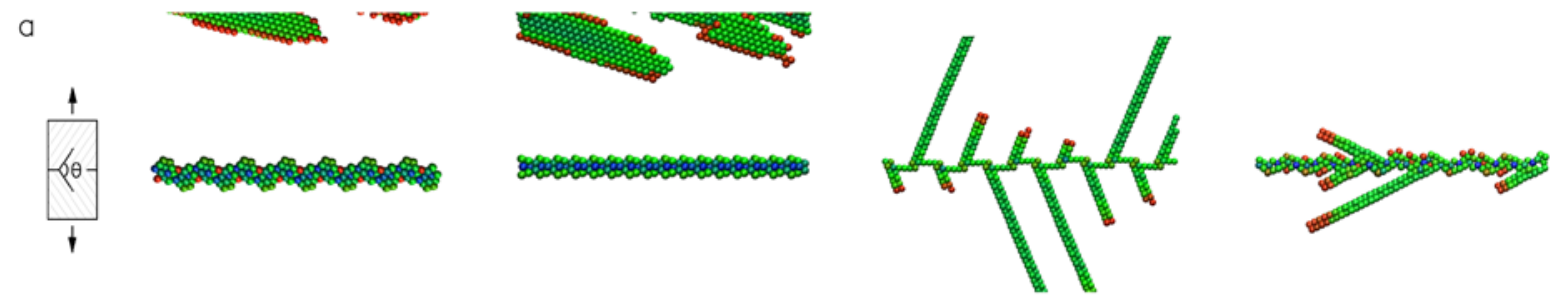

b

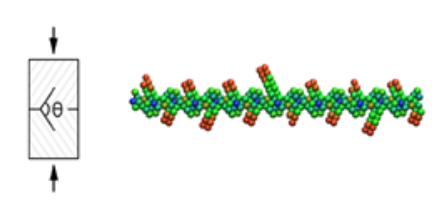

$\Sigma 19(116)$

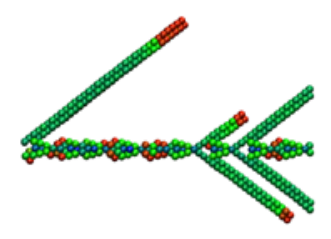

$\Sigma 27(115)$

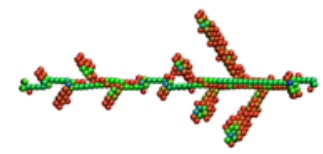

$\Sigma 33(554)$

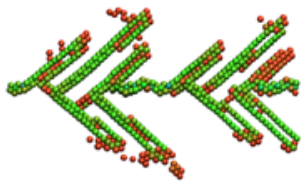

$\Sigma 33(441)$

Figure 4: Deformed atomic configurations of four typical symmetrical-tilt $<110>\mathrm{Ni}$ grain boundaries at the peak tensile (a) and compressive (b) strength corresponding to the instant of dislocation emission; structures are filtered based on the centro-symmetric parameter to display only non-FCC atoms, with dislocations colored in red and stacking faults colored in green. 
a

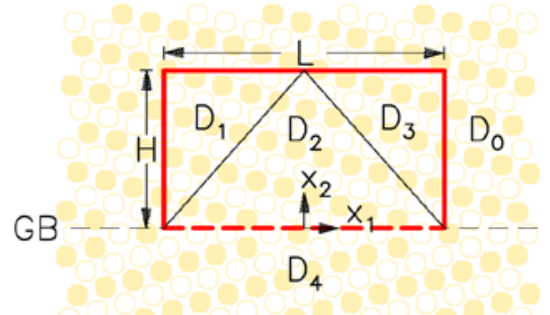

b

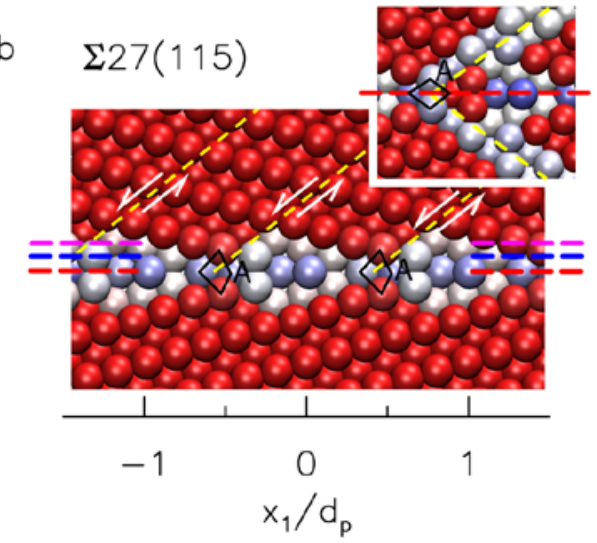

C
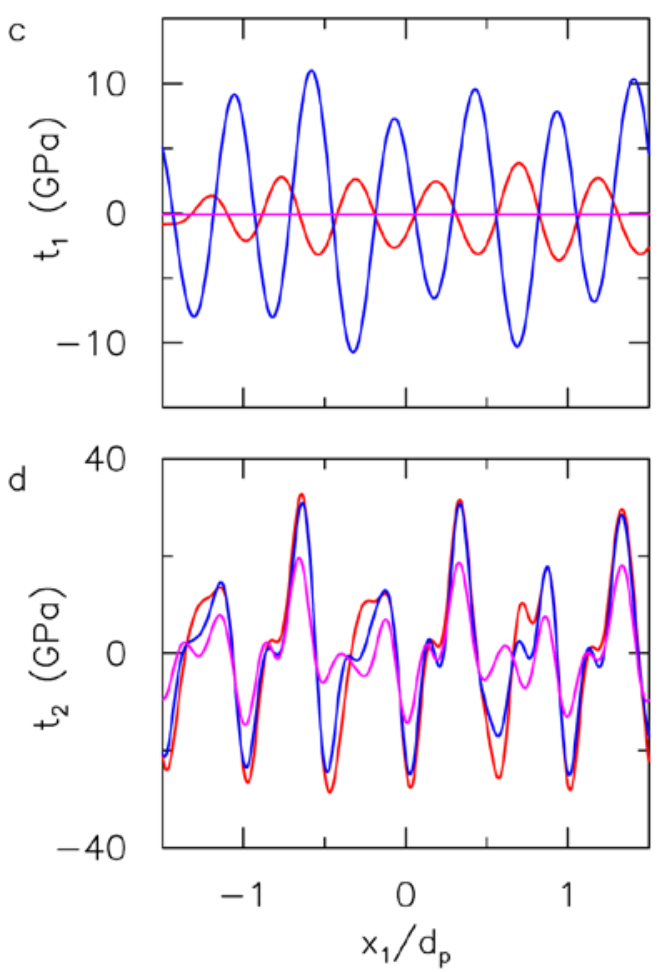

Figure 5: (a) Calculation domain of the field projection scheme to reconstruct the normal and shear tractions along the grain boundary (red dashed lines) from atomic stress information in the vicinity (enclosed by red lines). (b) Equilibrium atomic configuration of the $\Sigma 27(115)$ grain boundary; yellow dashed lines denote the active slip planes under uniaxial compression, with the deformed atomic configuration in the inset. (c,d) Field-projected shear $t_{1}$ (c) and normal $t_{2}$ (d) tractions along the corresponding colored dashed lines in (b). 

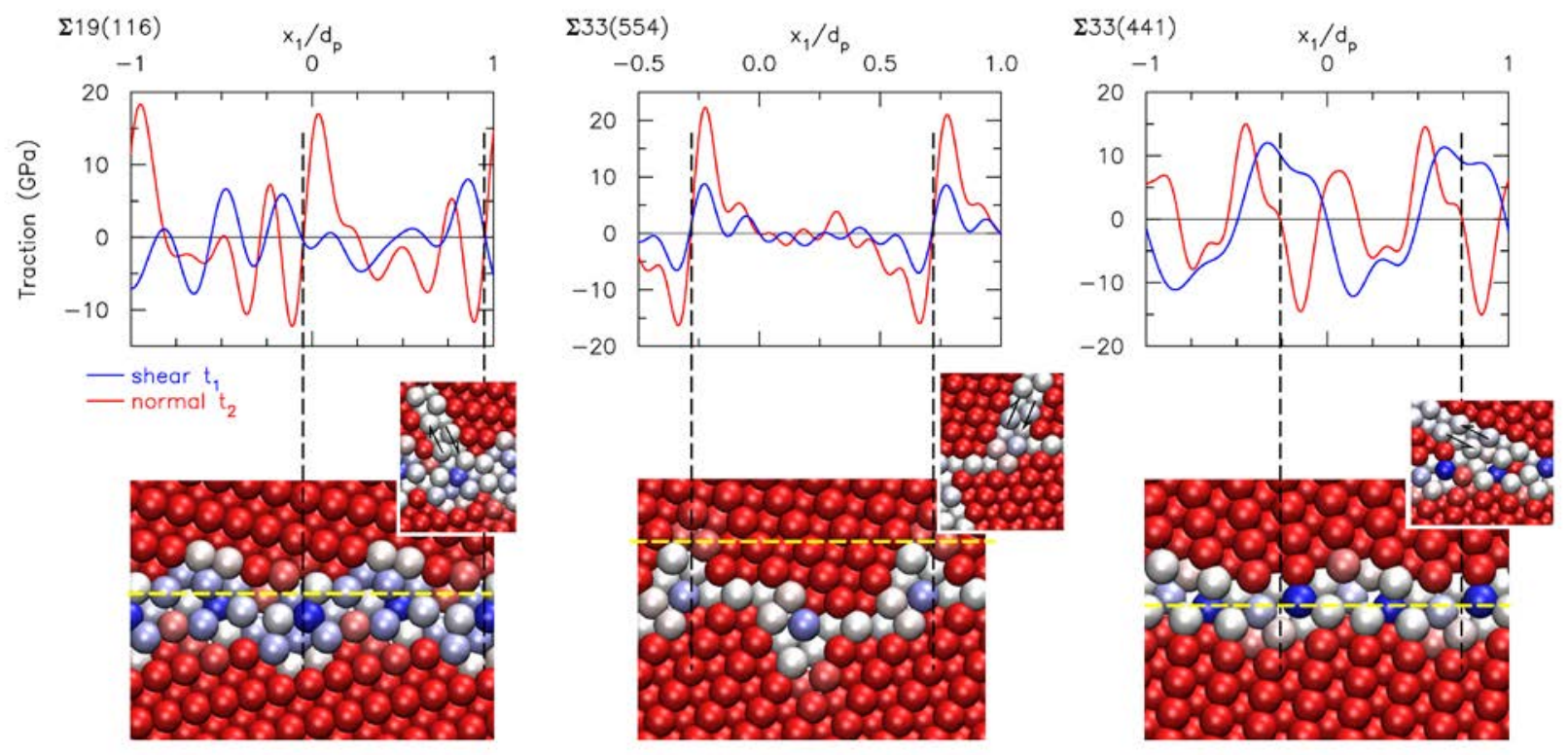

Figure 6: Field-projected shear $\left(t_{1}\right)$ and normal $\left(t_{2}\right)$ tractions along the yellow dashed lines of the equilibrium $\Sigma 19(116), \Sigma 33(554)$, and $\Sigma 33(441)$ grain boundary atomic configurations. Ready emission of dislocations, assisted by grain boundary tractions, initiating at the intersections between yellow and black dashed lines with corresponding deformed atomic configurations in the inset. 
a

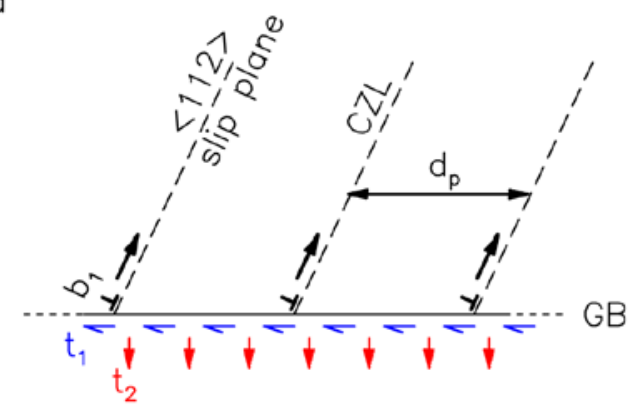

$\mathrm{b}$

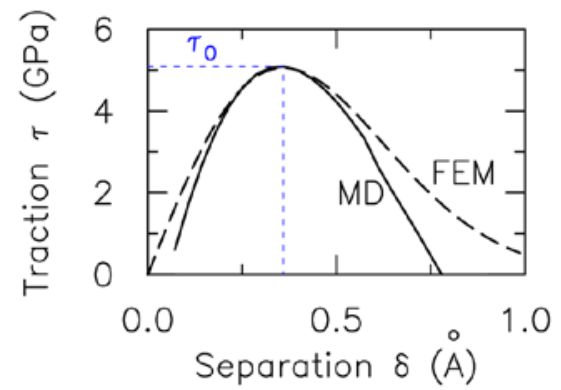

C
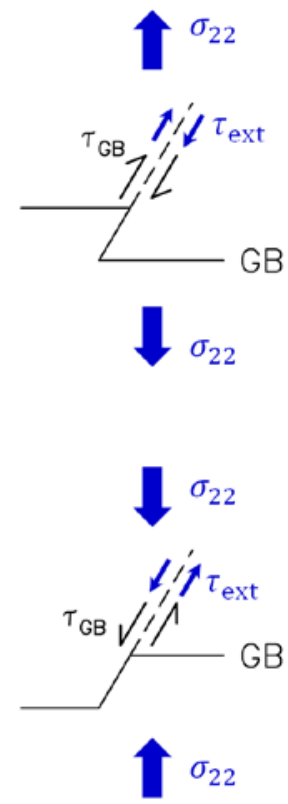

Figure 7: (a) Calculation of the resolved shear stress contribution $\tau_{\mathrm{GB}}$ from grain boundary shear $\left(t_{1}\right)$ and normal $\left(t_{2}\right)$ tractions from a finite elment half-space model with cohesive zone law implemented along the active slip-planes. (b) Cohesive zone law for shear-slip along the $<112>\{111\}$ slip system, as calculated from the gradient of the generalized stacking fault energy (GSFE) in MD, and fitted to an exponential model implented in FEM. (c) Illustration showing the ready emssion of dislocations only when $\tau_{\mathrm{GB}}$ acts in the same direction as the resolved shear stress contribution from external loading $\tau_{\text {ext }}$. 


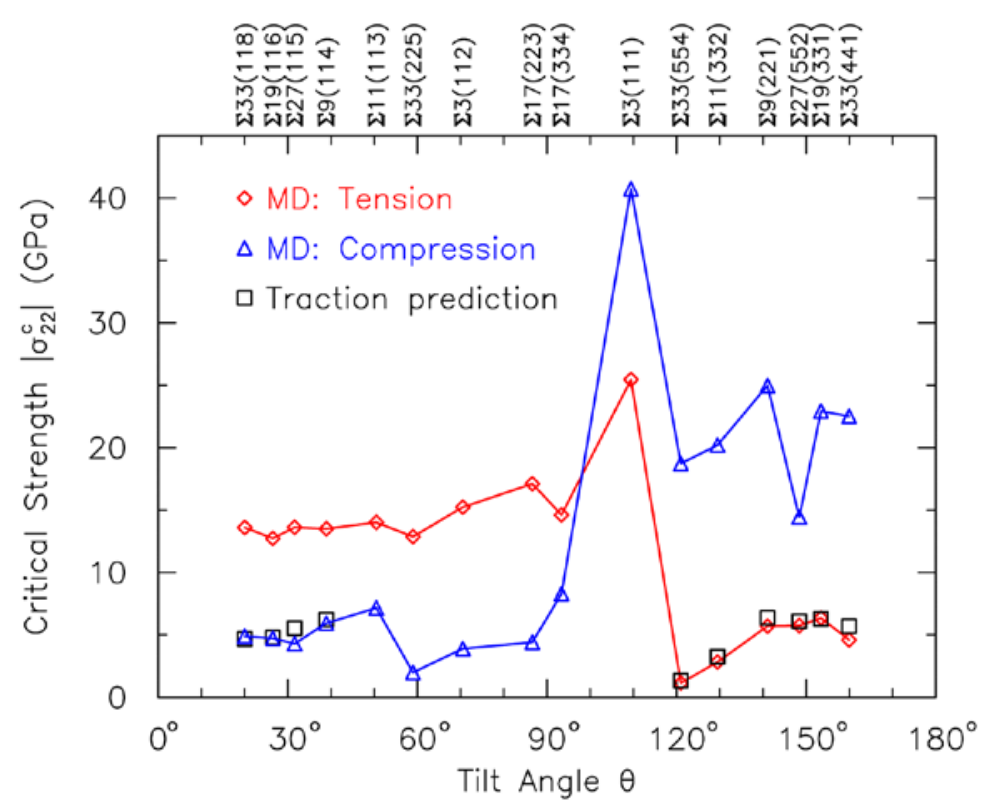

Figure 8: Summary of the critical tensile (red symbols) and compressive (blue symbols) strength of symmetrical-tilt $<110>\mathrm{Ni}$ grain boundaries from MD, and corresponding predictions (black symbols) from the traction signatures of the equilibrium grain boundary structures. 


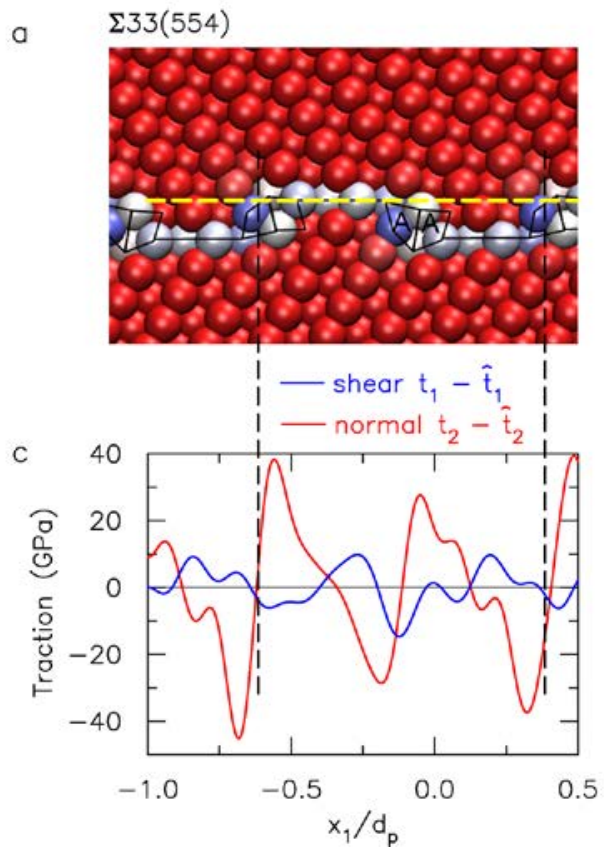

b
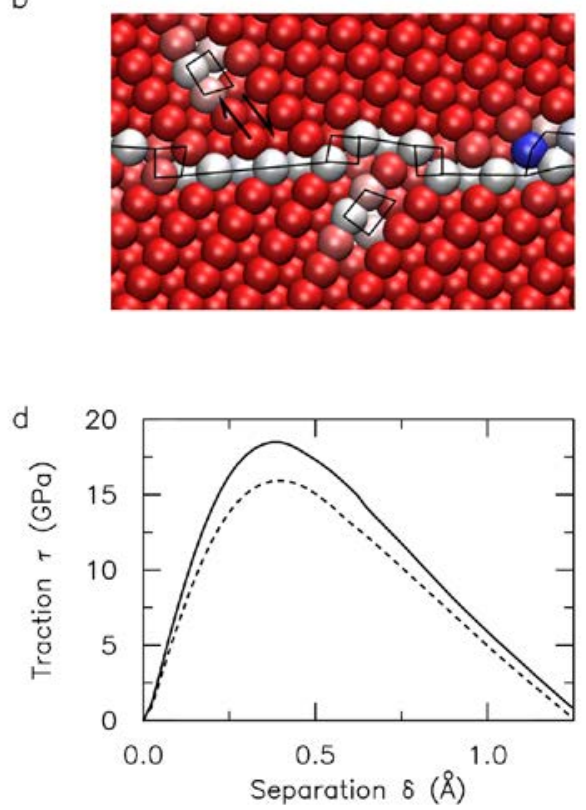

Figure 9: (a,b) Deformed atomic configurations of the $\Sigma 33(554)$ grain boundary under uniaxial compression, just prior to (a) and at the instant of (b) full dislocation emission. (c) Traction signatures along the deformed grain boundary structure corresponding to (a). (d) Traction separation laws for non-Schmid $\{001\}<110>$ dislocation slip in the absence of normal stresses to the $\{001\}$ slip-plane (dashed line), and at the stress-state corresponding to (a) (solid line). 

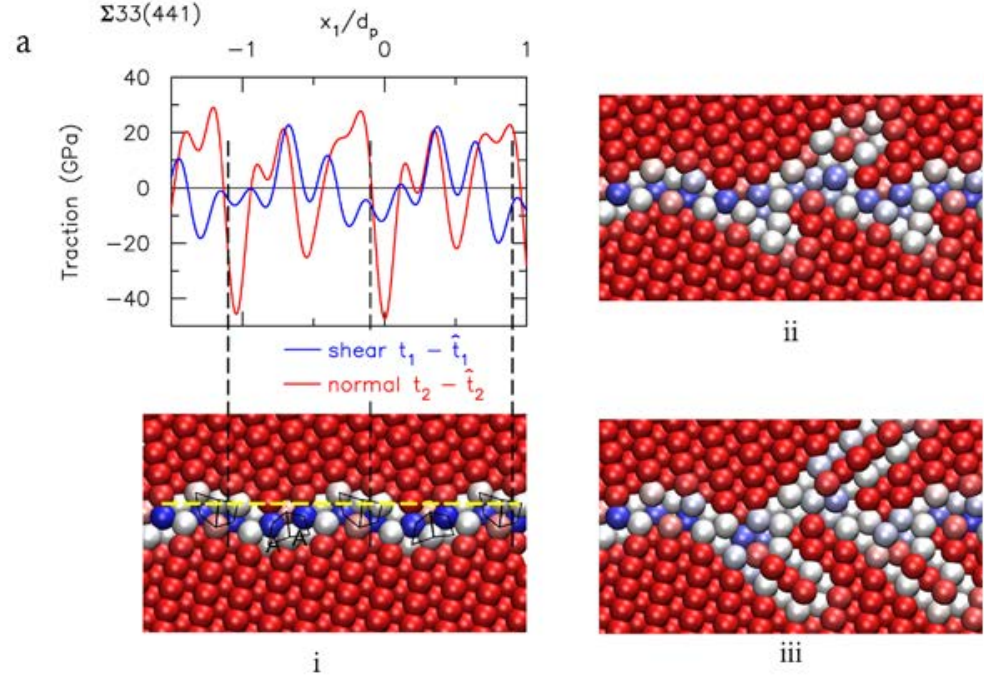

ii

$\mathrm{b}$
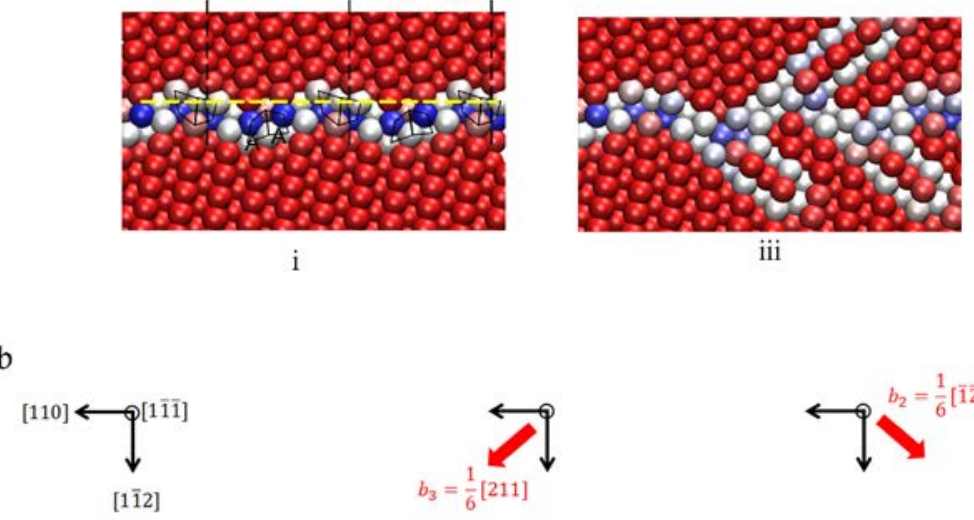

iii
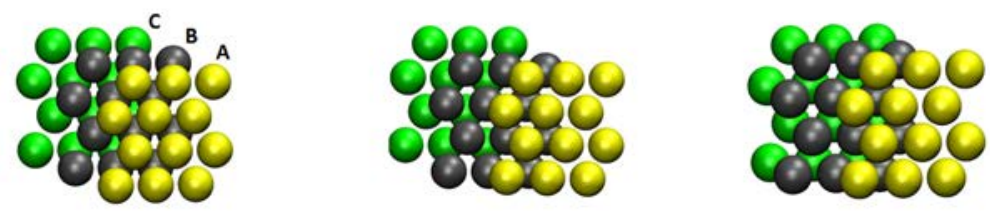

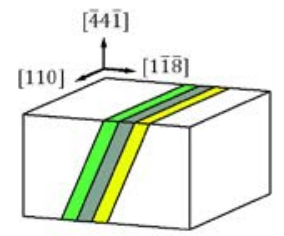

i

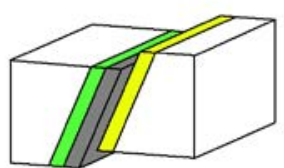

ii

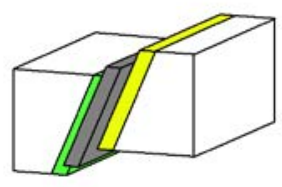

iii

Figure 10: (a) Deformed atomic configurations depicting sequence of out-of-plane partial dislocation emissions to form extrinsic stacking faults for $\Sigma 33(441)$ grain boundary under uniaxial compression, with traction signatures of the deformed grain boundary structure in (i) just prior to dislocation emission. (b) Top view and schematic representation of the stacking arrangements of ( $\overline{111}$ ) planes during the partial dislocation emission processes corresponding to (a). 